\title{
A combined clinical and biomarker approach to predict diuretic response in acute heart failure
}

\author{
Jozine M. ter Maaten ${ }^{1}$ Mattia A. E. Valente ${ }^{1} \cdot$ Marco Metra $^{2} \cdot$ Noemi Bruno $^{3}$. \\ Christopher M. O'Connor ${ }^{4} \cdot$ Piotr Ponikowski $^{5} \cdot$ John R. Teerlink ${ }^{6}$. \\ Gad Cotter $^{7} \cdot$ Beth Davison $^{7} \cdot$ John G. Cleland ${ }^{8} \cdot$ Michael M. Givertz $^{9}$ • \\ Daniel M. Bloomfield ${ }^{10} \cdot$ Howard C. Dittrich ${ }^{11}$ • Dirk J. van Veldhuisen ${ }^{1}$. \\ Hans L. Hillege ${ }^{1,12} \cdot$ Kevin Damman ${ }^{1} \cdot$ Adriaan A. Voors $^{1}$
}

Received: 2 June 2015/ Accepted: 20 July 2015/Published online: 18 August 2015

(C) The Author(s) 2015. This article is published with open access at Springerlink.com

\begin{abstract}
Background Poor diuretic response in acute heart failure is related to poor clinical outcome. The underlying mechanisms and pathophysiology behind diuretic resistance are incompletely understood. We evaluated a combined approach using clinical characteristics and biomarkers to predict diuretic response in acute heart failure (AHF).

Methods and results We investigated explanatory and predictive models for diuretic response-weight loss at day 4 per $40 \mathrm{mg}$ of furosemide-in 974 patients with AHF included in the PROTECT trial. Biomarkers, addressing multiple pathophysiological pathways, were determined at baseline and after $24 \mathrm{~h}$. An explanatory baseline biomarker model of a poor diuretic response included low potassium, chloride, hemoglobin, myeloperoxidase, and high blood urea nitrogen, albumin, triglycerides, ST2 and neutrophil gelatinase-associated lipocalin $\left(r^{2}=0.086\right)$. Diuretic response after $24 \mathrm{~h}$ (early diuretic response) was a strong predictor of diuretic response $(\beta=0.467, P<0.001$;
\end{abstract}

Electronic supplementary material The online version of this article (doi:10.1007/s00392-015-0896-2) contains supplementary material, which is available to authorized users.

Adriaan A. Voors

a.a.voors@umcg.nl

1 Department of Cardiology, University Medical Center Groningen, University of Groningen, Hanzeplein 1, 9713 GZ Groningen, The Netherlands

2 University of Brescia, Brescia, Italy

3 Department of Cardiovascular Sciences, "Sapienza" University of Rome, Rome, Italy

4 Duke University Medical Center, Durham, NC, USA

5 Clinical Military Hospital, Medical University, Wroclaw, Poland $r^{2}=0.523$ ). Addition of diuretic response after $24 \mathrm{~h}$ to biomarkers and clinical characteristics significantly improved the predictive model $\left(r^{2}=0.586, P<0.001\right)$. Conclusions Biomarkers indicate that diuretic unresponsiveness is associated with an atherosclerotic profile with abnormal renal function and electrolytes. However, predicting diuretic response is difficult and biomarkers have limited additive value. Patients at risk of poor diuretic response can be identified by measuring early diuretic response after $24 \mathrm{~h}$.

Keywords Diuretic response - Heart failure ·

Biomarkers · Prediction

$\begin{array}{ll}\text { Abbreviations } & \\ \text { AHF } & \text { Acute heart failure } \\ \text { BNP } & \text { Brain natriuretic peptide } \\ \text { BUN } & \text { Blood urea nitrogen } \\ \text { ESAM } & \begin{array}{l}\text { Endothelial cell-selective adhesion } \\ \text { molecule }\end{array} \\ & \text { Growth differentiation factor 15 } \\ \text { GDF-15 } & \text { Kidney injury molecule 1 } \\ \text { KIM-1 } & \text { Lymphotoxin beta receptor } \\ \text { LT } \beta \text { R } & \end{array}$

6 University of California at San Francisco and San Francisco Veterans Affairs Medical Center, San Francisco, CA, USA

7 Momentum Research, Durham, NC, USA

8 University of Hull, Kingston upon Hull, UK

9 Brigham and Women's Hospital, Boston, MA, USA

10 Merck Research Laboratories, Rahway, NJ, USA

11 University of Iowa Carver College of Medicine Cardiovascular Research Center, Iowa City, IA, USA

12 University Medical Center Groningen, Department of Epidemiology, University of Groningen, Groningen, The Netherlands 


\section{MPO Myeloperoxidase}

MR-proADM Mid regional pro-adrenomedullin

NGAL Neutrophil gelatinase-associated lipocalin

PROTECT Placebo-controlled randomized study of

the selective A1 adenosine receptor antagonist rolofylline for patients hospitalized with acute decompensated heart failure and volume overload to assess treatment effect on congestion and renal function

RAGE Receptor for advanced glycation endproducts

TNF-R1a Tumor necrosis factor receptor 1

\section{Introduction}

Treatment of acute heart failure (AHF) is primarily aimed at decongestion using diuretics. Suboptimal response to diuretics, or diuretic resistance, may reflect disease severity and is associated with impaired renal function and poor clinical outcome [1]. Recently, a definition for diuretic response was introduced, combining weight loss and diuretic dose, thus creating a quantitative indexed measure of diuretic response [2, 3]. Patients with a poor diuretic response had a significantly higher risk of post-discharge death or heart failure rehospitalization. Identification of patients with poor diuretic response or resistance early after hospital admission might lead to adaptation of treatment, potentially resulting in earlier relief of dyspnea, shorter length of hospital stay and-hypothetically - a reduced risk of hospital readmission early after discharge. Second, the pathophysiology behind the individual variation in diuretic response is not well understood. Valente et al. previously published the association of a poor diuretic response with more advanced heart failure, renal impairment, diabetes, and atherosclerotic disease [2]. Biomarkers might help in the early prediction of diuretic response during hospital admission, and may provide additional insights in the mechanisms underlying diuretic unresponsiveness. We, therefore, aimed to establish an explanatory and predictive model for diuretic response in acute heart failure using clinical characteristics and biomarkers from different pathophysiological domains.

\section{Methods}

\section{Study design and procedures}

The study population and design has previously been described in detail $[4,5]$. In short, 2033 patients hospitalized

for acute heart failure with mild to moderate renal dysfunction participated in the placebo-controlled randomized study of the selective A1 adenosine receptor antagonist rolofylline for patients hospitalized with acute decompensated heart failure and volume overload to assess treatment effect on congestion and renal function (PROTECT). The PROTECT study was a large, multicenter, phase III randomized clinical trial with neutral results. The trial was approved by the local ethics committee at each participating center. All patients provided written informed consent.

At baseline and after $24 \mathrm{~h}$, signs and symptoms of heart failure were assessed, as well as standard laboratory assessments. We selected 26 biomarkers based on their known association with outcome or severity of heart failure, renal function and atherosclerosis. Endothelial cell-selective adhesion molecule (ESAM), growth differentiation factor 15 (GDF-15), lymphotoxin beta receptor (LT $\beta R$ ), mesothelin, osteopontin, mid regional pro-adrenomedullin (MRproADM), receptor for advanced glycation endproducts (RAGE), soluble ST2, syndecan-1, and tumor necrosis factor alpha receptor 1 (TNF-R1a), were measured using sandwich enzyme-linked immunosorbent assays (ELISAs) on a Luminex ${ }^{\circledR}$ platform by Alere Inc., San Diego, Ca, USA. By use of competitive ELISAS on a Luminex ${ }^{\circledR}$ platform Angiogenin was measured. Finally, galectin-3, myeloperoxidase (MPO), and neutrophil gelatinase-associated lipocalin (NGAL) were measured using sandwich ELISAs on a microtiter plate. Two additional biomarkers, brain natriuretic peptide (BNP), and Kidney Injury molecule (KIM-1), were measured by single molecule counting technology by Erenna ${ }^{\circledR}$ Immunoassay System on a microtiter plate by Singulex Inc., Alameda, CA, USA.

\section{Definition of diuretic response and study population}

Diuretic response was defined as weight change on day 4 per $40 \mathrm{mg}$ of furosemide (or equivalent doses) administered from baseline to day 3 as described earlier [2]. Early diuretic response is defined as weight change after $24 \mathrm{~h}$ per $40 \mathrm{mg}$ of furosemide (or equivalent doses). Data on fluid intake, or urine output was not available. Of the 2033 included patients, 1288 patients had complete biomarker data available at baseline. A total of 1113 patients had additional complete biomarker data after $24 \mathrm{~h}$. Of these, 974 patients also had data of diuretic response available. This study, therefore, included a total study population of 974 patients. The selected subpopulation did not significantly differ from the excluded patients (supplementary Table 1).

\section{Statistical analysis}

The analyses were performed in the intention to treat population. Continuous variables are presented as 
mean \pm standard deviation or median with (interquartile range) when appropriate. Categorical values are presented as frequencies and percentages. Differences between groups were tested for significance with ANOVA (normal distribution) and Kruskal-Wallis (skewed distribution). A linear trend was statistically tested over quintiles of diuretic response, after checking for non-linear trends.

Uni- and multivariable linear regression analysis was performed with transformed values when necessary. Transformations were checked using multifractional polynomials. As a good diuretic response implicates lower values, standardized beta's need to be interpreted inverted, where a negative standardized beta means higher values are associated with a good diuretic response. Explanatory models were created based on statistical significance, whereas predictive models were selected on best fit [6]. Multivariable explanatory regression models, including all univariable variables with a $P$ value $<0.10$, were constructed via backward elimination and validated using bootstrap re-sampling with 1000 replicates. The models were tested for collinearity and checked by plotting residuals. Finally, an internal bootstrap with 1000 replicates of the selected models was performed, testing stability of these models. Spearman's rank correlation coefficient ( $r$ ) was used to assess the relation between predicted diuretic response and diuretic response after 4 days.

A two tailed $P$ value $<0.05$ was considered statistically significant. Analyses were performed using R: a Language and Environment for Statistical Computing, version 3.0.2. (R Foundation for Statistical Computing, Vienna, Austria).

\section{Results}

Biomarkers at baseline per quintile of diuretic response are presented in Table 1. Renal biomarkers, such as serum creatinine, blood urea nitrogen (BUN), and plasma NGAL, and atherosclerotic biomarkers (ESAM and LT $\beta$ R) showed significant trend over quintiles of diuretic response. Potassium, chloride, and sodium were significantly lower in patients with a poor response, whereas albumin and uric acid were significantly higher.

A similar pattern was observed for biomarker levels after $24 \mathrm{~h}$ (supplementary Table 2). Baseline characteristics of this population per quintile of diuretic response are presented in supplementary Table 3. In brief, poor responders had a lower blood pressure, more frequent diabetes and ischemic heart disease. An explanatory multimarker biomarker model included albumin, BUN, chloride, hemoglobin, MPO, NGAL, potassium, ST2, and triglycerides, but yielded only marginal explanatory value of diuretic response $\left(r^{2}=0.086\right)$. Higher chloride, hemoglobin, MPO and potassium levels were associated with a good diuretic response (Table 2). In addition, a good diuretic response was also associated with lower levels of albumin, BUN, NGAL, ST2, and triglycerides.

In addition, an explanatory model for biomarkers after $24 \mathrm{~h}$ included BUN, hemoglobin, MPO, sodium, ST2, and triglycerides $\left(r^{2}=0.082\right)$. Again, higher hemoglobin, MPO, and sodium levels, and lower levels of BUN, ST2, and triglycerides were associated with a good diuretic response (supplementary Table 4). Finally, an explanatory clinical baseline model (Table 3) showed that good diuretic response was associated with higher systolic blood pressure, higher weight and JVP, less frequent history of Diabetes Mellitus, PCI, COPD, beta blocker, and metolazone use, and more spironolactone use and randomized rolofylline treatment $\left(r^{2}=0.134\right)$.

Exploration of a model that contained variables $24 \mathrm{~h}$ after randomization identified early diuretic response (after $24 \mathrm{~h})$ as a strong predictor of good diuretic response (univariable $\beta=0.467, P<0.001 ; r^{2}=0.523$ ) at 4 days. In Fig. 1 median diuretic response and interquartile ranges after 4 days are plotted per quintile of early diuretic response from, respectively, good (quintile 1) to poor response (quintile 5). This figure shows that a poor early response had reasonable consistency with diuretic response values after 4 days. Prediction of diuretic response at day 4 based on early diuretic response alone showed a strong correlation $(r=0.723, P<0.001)$. The scatter plot of predicted response (based on the early response after $24 \mathrm{~h}$ ) and measured diuretic response on day 4 is shown in Fig. 2. Out of 974 patients, 98 patients $(10.1 \%)$ had a good early diuretic response after $24 \mathrm{~h}$ (>median) and a poor response on day 4 ( $\leq$ median). Clinical characteristics and biomarkers revealed no important differences between this group and other groups based on response after $24 \mathrm{~h}$ and on day 4.

Based on these findings, we explored a 24 -h predictive model for diuretic response (Table 4). In addition to early diuretic response (after $24 \mathrm{~h}$ ), BUN at baseline, change in BUN (24 h-baseline), systolic blood pressure, weight at baseline and randomized rolofylline treatment, as well as a history of COPD, Diabetes Mellitus, PCI, and JVP were included in the model. The use of spironolactone, and beta blockers, baseline potassium, triglycerides, chloride, and MPO, and ST2, and hemoglobin after $24 \mathrm{~h}$ further provided a significant improvement of fit $\left(r^{2}=0.586, P<0.001\right)$. Internal bootstrapping gave similar results.

\section{Discussion}

Poor response to diuretics in patients admitted with acute heart failure is a clinical problem associated with high morbidity and mortality. Using a panel of clinical and 


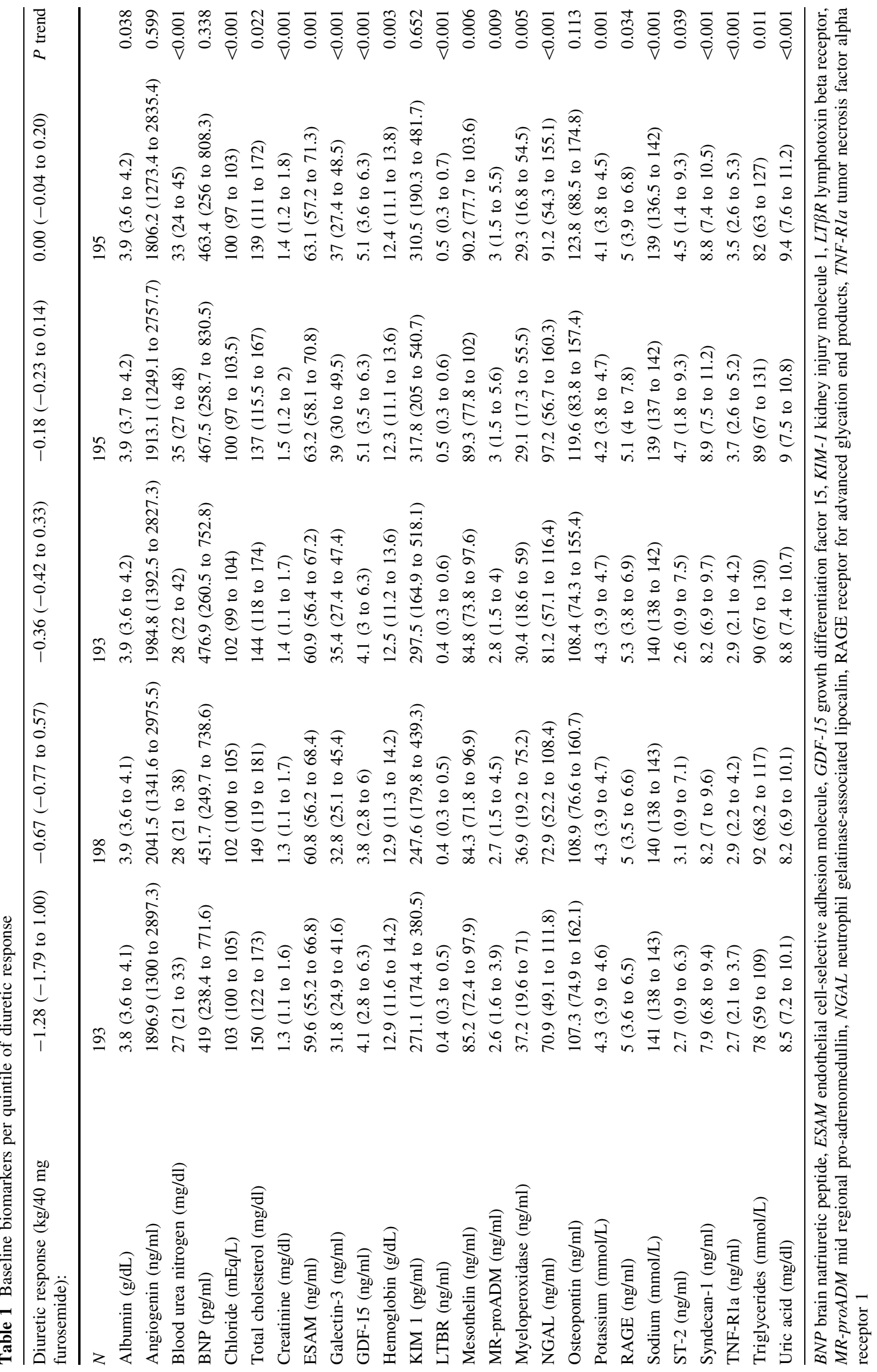


Table 2 Explanatory

biomarker baseline model

\begin{tabular}{lclrr}
\hline Variable & Beta coefficient & $95 \%$ CI & $T$ value & $P$ value \\
\hline Albumin (per SD) & 0.072 & 0.03 to 0.12 & 3.045 & 0.002 \\
Log blood urea nitrogen (per SD) & 0.076 & 0.02 to 0.13 & 2.797 & 0.005 \\
Chloride (per SD) & -0.066 & -0.11 to -0.02 & -2.772 & 0.006 \\
Hemoglobin (per SD) & -0.058 & -0.11 to -0.01 & -2.308 & 0.021 \\
Myeloperoxidase (per SD) & -0.060 & -0.11 to -0.01 & -2.490 & 0.013 \\
NGAL (per SD) & 0.064 & 0.01 to 0.12 & 2.307 & 0.021 \\
Potassium (per SD) & -0.103 & -0.15 to -0.06 & -4.333 & $<0.001$ \\
ST2 (per SD) & 0.054 & 0.01 to 0.10 & 2.213 & 0.027 \\
Triglycerides (per SD) & 0.060 & 0.02 to 0.10 & 2.814 & 0.005 \\
\hline
\end{tabular}

$r^{2}=0.086$

$N G A L$ neutrophil gelatinase-associated lipocalin
Table 3 Explanatory clinical baseline model

\begin{tabular}{lccrr}
\hline Variable & Beta coefficient & $95 \%$ CI & $T$ value & $P$ value \\
\hline Weight (per SD) & -0.089 & -0.13 to -0.04 & -3.781 & $<0.001$ \\
Systolic blood pressure (per SD) & -0.082 & -0.13 to -0.04 & -3.648 & $<0.001$ \\
Rolofylline treatment & -0.138 & -0.23 to -0.05 & -2.999 & 0.003 \\
Jugular venous pressure & -0.107 & -0.19 to -0.02 & -2.386 & 0.017 \\
Diabetes mellitus & 0.159 & 0.07 to 0.25 & 3.490 & 0.001 \\
PCI & 0.181 & 0.08 to 0.28 & 3.558 & $<0.001$ \\
COPD & 0.127 & 0.02 to 0.23 & 2.294 & 0.022 \\
Beta blocker & 0.207 & 0.10 to 0.31 & 3.963 & $<0.001$ \\
Spironolactone & -0.131 & -0.22 to -0.04 & -2.869 & 0.004 \\
Metolazone & 0.317 & 0.14 to 0.50 & 3.432 & 0.001 \\
\hline$r^{2}=0.134$ & & & &
\end{tabular}

$P C I$ percutaneous coronary intervention, $C O P D$ chronic obstructive pulmonary disease biochemical markers, we aimed to determine a non-response profile for two reasons. First, it is of clinical relevance to predict patients at risk, to initiate early alternative therapies [7]. Second, a distinct clinical or biochemical profile might provide more information about a pathophysiological mechanism behind diuretic unresponsiveness.

\section{Identification of patients at risk of poor diuretic response}

Clinical characteristics and biomarkers fall short in predicting diuretic response. However, assessment of early diuretic response after $24 \mathrm{~h}$ allows the clinician to identify patients at risk of diuretic resistance shortly after hospital admission. Although it is probably not surprising that diuretic response at day 4 is predicted by early diuretic response after only $24 \mathrm{~h}$, it provides important clinical application. This readily applicable metric can be used daily in all patients hospitalized for acute heart failure. Implementation of this metric in clinical practice will identify both patients with favorable diuretic response and patients with diuretic resistance early on during hospitalization. Once either of these patients are identified, treatment strategies may be adapted. Alternative and sometimes more aggressive strategies can be explored in patients with significant unresponsiveness with a great risk of adverse outcome, as these patients are more likely to benefit from alternative therapies. Several strategies can be considered. One of these is combination diuretic therapy-addition of a thiazide diuretic or a mineralocorticoid receptor antagonist at natriuretic doses - can help overcome diuretic resistance and improve natriuresis [8-11]. Other alternatives are for instance, adding dopamine or switching to ultrafiltration [7]. Although the larger all-comer trials were neutral for these therapies, both of these approaches have been insufficiently investigated in truly unresponsive, diuretic resistant patients. Our analyses also showed that randomized allocation to rolofylline, an adenosine A-1 antagonist, was associated with a good diuretic response. This might suggest that, in specific subpopulations, rolofylline may help overcome poor diuretic response. Whether this will 


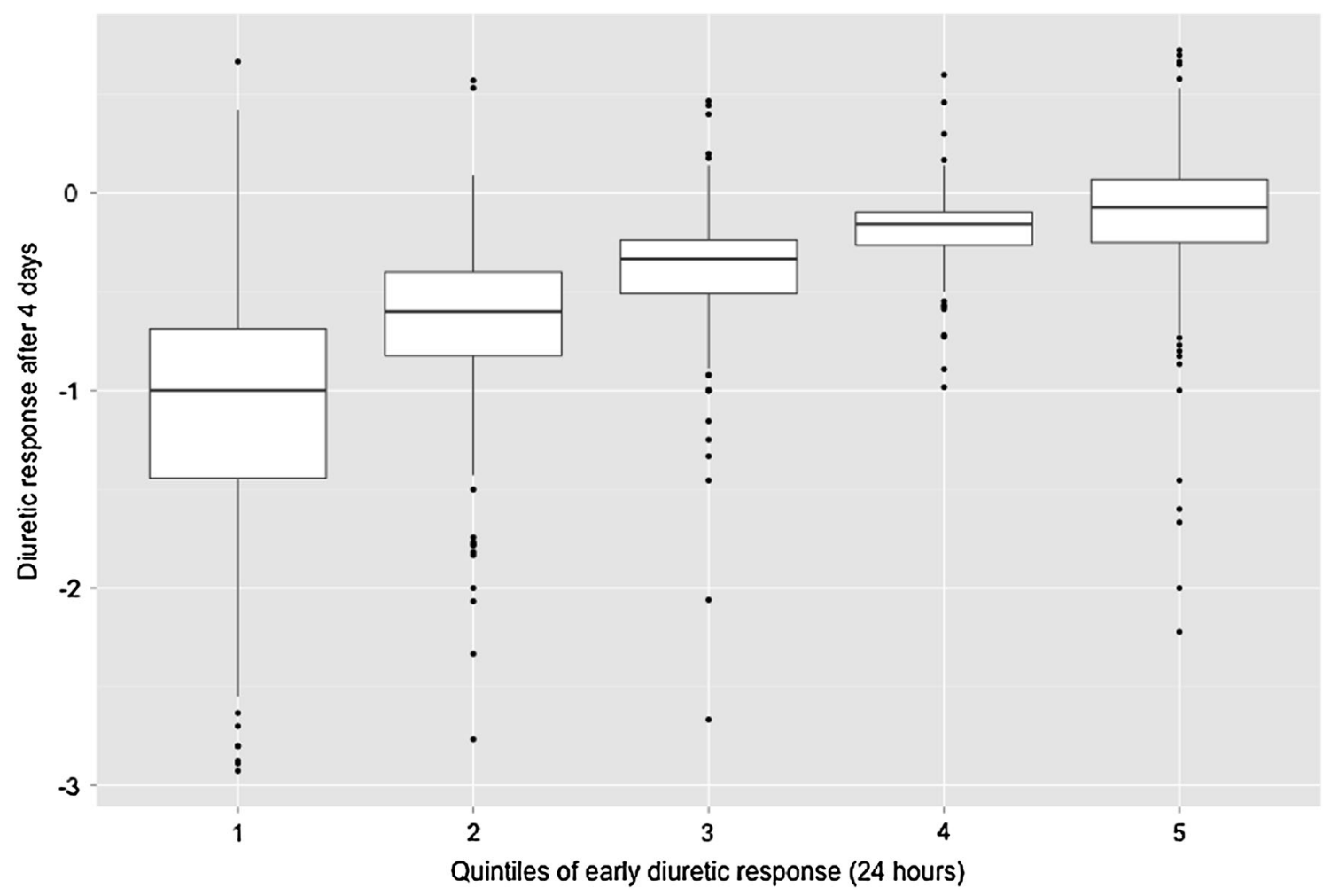

Fig. 1 Diuretic response after 4 days per quintile of diuretic response after $24 \mathrm{~h}$

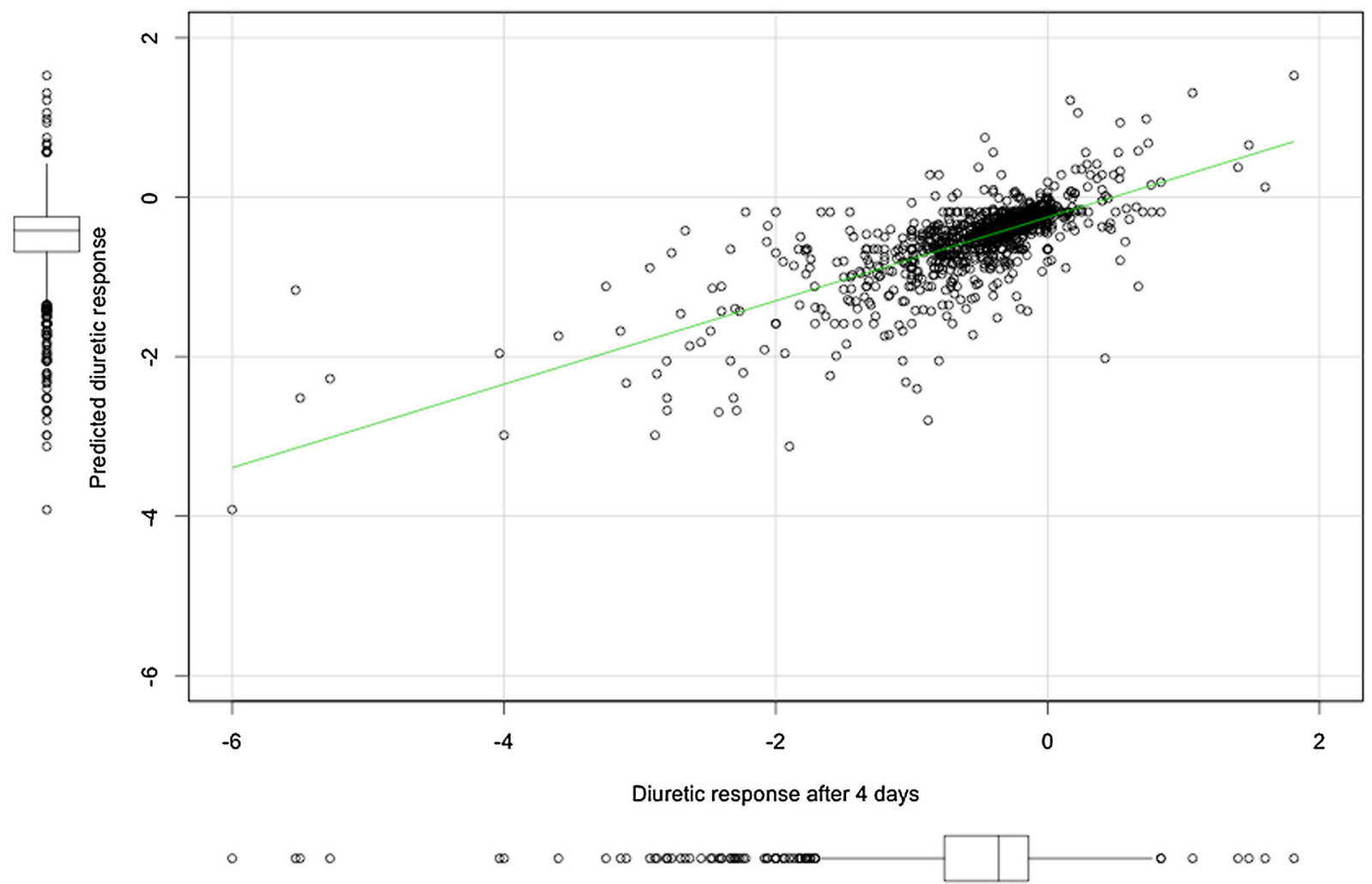

Fig. 2 Scatter plot of predicted response and calculated diuretic response after 4 days 
Table 424 hour diuretic response prediction model

\begin{tabular}{|c|c|c|c|c|}
\hline Variable & Beta coefficient & $95 \% \mathrm{CI}$ & $T$ value & $P$ value \\
\hline Early diuretic response (after 24 h) (per SD) & 0.479 & 0.45 to 0.51 & 28.052 & $<0.001$ \\
\hline Systolic blood pressure (per SD) & -0.068 & -0.10 to -0.04 & -4.208 & $<0.001$ \\
\hline Change in blood urea nitrogen ( $24 \mathrm{~h}-$ baseline) (per SD) & 0.055 & 0.02 to 0.09 & 3.403 & 0.001 \\
\hline Potassium at baseline (per SD) & -0.053 & -0.08 to -0.02 & -3.207 & 0.001 \\
\hline COPD & 0.111 & 0.03 to 0.19 & 2.869 & 0.004 \\
\hline Triglycerides at baseline (per SD) & 0.038 & 0.01 to 0.07 & 2.646 & 0.008 \\
\hline Diabetes mellitus & 0.079 & 0.01 to 0.14 & 2.397 & 0.017 \\
\hline Beta blocker & 0.088 & 0.02 to 0.16 & 2.393 & 0.017 \\
\hline Weight (per SD) & -0.037 & -0.07 to -0.01 & -2.279 & 0.023 \\
\hline Spironolactone & -0.073 & -0.14 to -0.01 & -2.262 & 0.024 \\
\hline Jugular venous pressure & -0.072 & -0.13 to -0.01 & -2.31 & 0.021 \\
\hline PCI & 0.074 & 0.00 to 0.14 & 2.059 & 0.040 \\
\hline Log blood urea nitrogen at baseline (per SD) & 0.033 & 0.00 to 0.07 & 1.920 & 0.055 \\
\hline Rolofylline treatment & -0.060 & -0.12 to 0.00 & -1.883 & 0.060 \\
\hline ST2 after $24 \mathrm{~h}$ (per SD) & 0.029 & 0.00 to 0.06 & 1.778 & 0.076 \\
\hline Hemoglobin after $24 \mathrm{~h}$ (per SD) & -0.026 & -0.06 to 0.01 & -1.586 & 0.113 \\
\hline Chloride at baseline (per SD) & -0.021 & -0.05 to 0.01 & -1.274 & 0.203 \\
\hline Myeloperoxidase at baseline (per SD) & -0.019 & -0.05 to 0.01 & -1.152 & 0.250 \\
\hline
\end{tabular}

$r^{2}=0.586$

$C O P D$ chronic obstructive pulmonary disease, $P C I$ percutaneous coronary intervention

also improve outcome is unknown. Future studies should aim to investigate the effects of alternative strategies on relieve of dyspnea and clinical outcome in patients admitted for acute heart failure.

\section{Predictors of diuretic response}

Many clinical variables and biomarkers are related to diuretic response. Our analyses show that a poor diuretic response is strongly associated with renal and atherosclerotic biomarkers, like creatinine, NGAL, ESAM and LT $\beta$ R. The clinical characteristics, previously described by Valente et al. provided the same results in this smaller subset of patients from the PROTECT cohort [2]. Atherosclerotic characteristics as well as higher levels of novel atherosclerotic biomarkers like ESAM and LT $\beta$ R were associated with a poor response. In the Dallas Heart study ESAM was associated with subclinical atherosclerosis; while LT $\beta R$ also associated with multiple signs of atherosclerosis in this study, confirming that a link between these markers and poor diuretic response could be pointing towards a phenotype with atherosclerotic properties [12, 13].

Interestingly, renal biomarkers, such as creatinine, BUN and NGAL, were more abnormal over increasing quintiles of diuretic response and were significant predictors of diuretic response. Renal tubular function is of key importance for diuretic efficacy [14]. The finding that higher levels of plasma NGAL were associated with a poor diuretic response supports this. However, as plasma KIM-1 was not significantly associated with diuretic response; the question remains whether circulating NGAL and KIM-1 levels both reflect tubular function [15]. A higher creatinine and BUN level was associated with a poor response to diuretic treatment. Ferreira et al. previously identified plasma urea as a predictor of slower diuretic response [16]. In our study, an increase in BUN is also predictive of poor diuretic response, suggesting not only baseline values but also worsening of renal function is of influence on diuretic response. Both, renin-angiotensin-aldosterone system and sympathetic nervous system activation cause a flow-dependent passive resorption of urea in the distal tubule, caused by increased sodium and water resorption in the proximal tubule $[17,18]$. This consequently results in diminished distal flow and increased reabsorption. Elevated BUN levels, therefore, indicate a kidney working actively to retain water and sodium. This could be one of the reasons for the far greater increase in BUN compared with serum creatinine with poorer diuretic response. In addition, loop diuretics need to be actively secreted by the organic anion transporter in the proximal tubule to arrive at their site of action at the luminal side of the tubule $[19,20]$. Organic anions, like uric acid, competitively bind this receptor, thus causing diminished diuretic availability [21, 22].

Several electrolytes, like potassium, sodium and chloride showed associations with diuretic response. Interestingly, higher chloride levels were associated with a better 
diuretic response. High chloride levels have been shown to reduce renin release and increase blood pressure [23]. In addition, loop diuretics inhibit the reabsorption of chloride in the loop of Henle. A higher chloride level may, therefore, slightly suppress the renin-angiotensin-aldosterone system, hence possibly increasing renal perfusion and sodium reabsorption, and provide a less depleted chloride level during diuretic treatment. Similarly, a low potassium was associated with a poor diuretic response, likely due to its co-transporter function.

Finally, MPO, ST2 and NGAL were the only 'novel' biomarkers in the multivariable models of diuretic response. Interestingly, both ST2 and MPO are thought to be associated with a pro-inflammatory state. Higher MPO levels are associated with more advanced heart failure and adverse outcome in chronic heart failure patients [24]. In patients with acute coronary syndrome, pre-admission treatment with statins, beta blockers or ACE inhibitors reduced MPO levels [25]. In this study we paradoxically found an association between low MPO levels and poor diuretic response. Higher ST2 levels have been found in chronic kidney disease patients and correlated with disease severity [26]. Addition of ST2 to BNP in acute heart failure patients has been shown to improve prognostic accuracy [27].

\section{Limitations}

This study is a retrospective analysis of a randomized clinical trial. Unfortunately, not all patients had complete biomarker data available at baseline and after $24 \mathrm{~h}$, creating a selected subpopulation used for these analyses. In addition, these analyses are data driven and causality cannot be proven. The results of this study need to be validated in a different population. Research assays to MR-proADM, galectin-3, and ST2 were developed by Alere, and have not been standardized to the commercialized assays used in research or in clinical use. The extent to which each Alere assay correlates with the commercial assay is not fully characterized. Information on fluid intake, urine output or net fluid balance was not collected in the PROTECT database. We were unfortunately not able to compare diuretic response based on weight loss to other metrics, for instance based on urine excretion or net fluid loss.

\section{Conclusions}

Biomarkers indicate that poor diuretic response is associated with a profile of atherosclerosis, glomerular and tubular renal dysfunction and abnormal electrolytes. These markers were of limited clinical use to predict diuretic response at hospital admission for acute heart failure.
Patients at risk of diuretic resistance can be identified by measuring diuretic response after $24 \mathrm{~h}$.

Acknowledgments The PROTECT trial was supported by NovaCardia, a subsidiary of Merck. Alere and Singulex kindly provided assays and performed biomarker measurements.

\section{Compliance with ethical standards}

Disclosures J.M.tM., M.A.E.V., N.B., H.L.H., and K.D. have nothing to disclose. A.A.V. has received speaker and consultancy fees from Merck and NovaCardia. D.J.V.V. has received Board Membership fees from Amgen, Biocontrol, Johnson \& Johnson, Novartis, Sorbent and Vifor. C.M.O.C. is a consultant to Merck. M.M. has received honoraria and reimbursements from NovaCardia, sponsors of the study, and from Merck, that purchased the rights to rolofylline after completion of the PROTECT pilot study. P.P. has received honoraria from Merck. J.R.T. has received research funds and consulting fees from Merck, the producer of rolofylline for the conduct of this study and has also received research funds and consulting fees from Abbott, Amgen, Biogen Idec, Corthera, Cytokinetics, Johnson and Johnson/Scios, Novartis, Relypsa and Solvay for research in related areas. G.C. and B.D. are employees of Momentum Research Inc, which was contracted to perform work on the project by Merck \& Co, Inc. J.G.C. was on the Steering Committee for the study, served on the Advisory Board for MSD, and received payments for both. M.M.G. has received institutional research support and served on a scientific Advisory Board for Merck. D.M.B. is an employee of Merck. H.C.D was an employee of NovaCardia and a consultant to Merck.

Open Access This article is distributed under the terms of the Creative Commons Attribution 4.0 International License (http://crea tivecommons.org/licenses/by/4.0/), which permits unrestricted use, distribution, and reproduction in any medium, provided you give appropriate credit to the original author(s) and the source, provide a link to the Creative Commons license, and indicate if changes were made.

\section{References}

1. Ronco C, McCullough P, Anker SD, Anand I, Aspromonte N, Bagshaw SM, Bellomo R, Berl T, Bobek I, Cruz DN, Daliento L, Davenport A, Haapio M, Hillege H, House AA, Katz N, Maisel A, Mankad S, Zanco P, Mebazaa A, Palazzuoli A, Ronco F, Shaw A, Sheinfeld G, Soni S, Vescovo G, Zamperetti N, Ponikowski P, Acute Dialysis Quality (ADQI) consensus group (2010) Cardiorenal syndromes: report from the consensus conference of the acute dialysis quality initiative. Eur Heart J 31:703-711

2. Valente MA, Voors AA, Damman K, Van Veldhuisen DJ, Massie BM, O'Connor CM, Metra M, Ponikowski P, Teerlink JR, Cotter G, Davison B, Cleland JG, Givertz MM, Bloomfield DM, Fiuzat M, Dittrich HC, Hillege HL (2014) Diuretic response in acute heart failure: clinical characteristics and prognostic significance. Eur Heart J 35:1284-1293

3. Voors AA, Davison BA, Teerlink JR, Felker GM, Cotter G, Filippatos G, Greenberg BH, Pang PS, Levin B, Hua TA, Severin T, Ponikowski P, Metra M, RELAX-AHF Investigators (2014) Diuretic response in patients with acute decompensated heart failure: characteristics and clinical outcome-an analysis from RELAX-AHF. Eur J Heart Fail 16:1230-1240 
4. Weatherley BD, Cotter G, Dittrich HC, DeLucca P, Mansoor GA, Bloomfield DM, Ponikowski P, O'Connor CM, Metra M, Massie BM, PROTECT Steering Committee, Investigators, and Coordinators (2010) Design and rationale of the PROTECT study: a placebo-controlled randomized study of the selective A1 adenosine receptor antagonist rolofylline for patients hospitalized with acute decompensated heart failure and volume overload to assess treatment effect on congestion and renal function. J Card Fail 16:25-35

5. Massie BM, O'Connor CM, Metra M, Ponikowski P, Teerlink JR, Cotter G, Weatherley BD, Cleland JG, Givertz MM, Voors A, DeLucca P, Mansoor GA, Salerno CM, Bloomfield DM, Dittrich HC, PROTECT Investigators and Committees (2010) Rolofylline, an adenosine A1-receptor antagonist, in acute heart failure. N Engl J Med 363:1419-1428

6. Shmueli G (2010) To explain or to predict? Stat Sci 25:289-310

7. Ter Maaten JM, Valente MA, Damman K, Hillege HL, Navis G, Voors AA (2015) Diuretic response in acute heart failurepathophysiology, evaluation, and therapy. Nat Rev Cardiol 12:184-192

8. Kunau RT Jr, Weller DR, Webb HL (1975) Clarification of the site of action of chlorothiazide in the rat nephron. J Clin Invest 56:401-407

9. Channer KS, McLean KA, Lawson-Matthew P, Richardson M (1994) Combination diuretic treatment in severe heart failure: a randomised controlled trial. Br Heart J 71:146-150

10. van Vliet AA, Donker AJ, Nauta JJ, Verheugt FW (1993) Spironolactone in congestive heart failure refractory to high-dose loop diuretic and low-dose angiotensin-converting enzyme inhibitor. Am J Cardiol 71:21A-28A

11. Ferreira JP, Santos M, Almeida S, Marques I, Bettencourt P, Carvalho H (2014) Mineralocorticoid receptor antagonism in acutely decompensated chronic heart failure. Eur J Intern Med 25:67-72

12. Rohatgi A, Owens AW, Khera A, Ayers CR, Banks K, Das SR, Berry JD, McGuire DK, de Lemos JA (2009) Differential associations between soluble cellular adhesion molecules and atherosclerosis in the Dallas Heart Study: a distinct role for soluble endothelial cell-selective adhesion molecule. Arterioscler Thromb Vasc Biol 29:1684-1690

13. Owens AW, Matulevicius S, Rohatgi A, Ayers CR, Das SR, Khera A, McGuire DK, de Lemos JA (2010) Circulating lymphotoxin beta receptor and atherosclerosis: observations from the Dallas Heart Study. Atherosclerosis 212:601-606

14. Cleland JG, Coletta A, Witte K (2006) Practical applications of intravenous diuretic therapy in decompensated heart failure. Am J Med 119:S26-S36

15. Shrestha K, Shao Z, Singh D, Dupont M, Tang WH (2012) Relation of systemic and urinary neutrophil gelatinase-associated lipocalin levels to different aspects of impaired renal function in patients with acute decompensated heart failure. Am J Cardiol 110:1329-1335

16. Ferreira JP, Santos M, Almeida S, Marques I, Bettencourt P, Carvalho H (2013) Tailoring diuretic therapy in acute heart failure: insight into early diuretic response predictors. Clin Res Cardiol 102:745-753

17. Kazory A (2010) Emergence of blood urea nitrogen as a biomarker of neurohormonal activation in heart failure. Am J Cardiol 106:694-700

18. Schrier RW (2008) Blood urea nitrogen and serum creatinine: not married in heart failure. Circ Heart Fail 1:2-5

19. Uwai Y, Saito H, Hashimoto Y, Inui KI (2000) Interaction and transport of thiazide diuretics, loop diuretics, and acetazolamide via rat renal organic anion transporter rOAT1. J Pharmacol Exp Ther 295:261-265

20. Kim EJ, Lee MG (2001) Pharmacokinetics and pharmacodynamics of intravenous bumetanide in mutant Nagase analbuminemic rats: importance of globulin binding for the pharmacodynamic effects. Biopharm Drug Dispos 22:147-156

21. Sweet DH, Bush KT, Nigam SK (2001) The organic anion transporter family: from physiology to ontogeny and the clinic. Am J Physiol Renal Physiol 281:F197-F205

22. Krick W, Wolff NA, Burckhardt G (2000) Voltage-driven p-aminohippurate, chloride, and urate transport in porcine renal brush-border membrane vesicles. Pflugers Arch 441:125-132

23. Kotchen TA, Luke RG, Ott CE, Galla JH, Whitescarver S (1983) Effect of chloride on renin and blood pressure responses to sodium chloride. Ann Intern Med 98:817-822

24. Tang WH, Tong W, Troughton RW, Martin MG, Shrestha K, Borowski A, Jasper S, Hazen SL, Klein AL (2007) Prognostic value and echocardiographic determinants of plasma myeloperoxidase levels in chronic heart failure. J Am Coll Cardiol 49:2364-2370

25. Ndrepepa G, Braun S, Schomig A, Kastrati A (2011) Impact of therapy with statins, beta-blockers and angiotensin-converting enzyme inhibitors on plasma myeloperoxidase in patients with coronary artery disease. Clin Res Cardiol 100:327-333

26. Bao YS, Na SP, Zhang P, Jia XB, Liu RC, Yu CY, Mu SH, Xie RJ (2012) Characterization of interleukin-33 and soluble ST2 in serum and their association with disease severity in patients with chronic kidney disease. J Clin Immunol 32:587-594

27. Frioes F, Lourenco P, Laszczynska O, Almeida PB, Guimaraes JT, Januzzi JL, Azevedo A, Bettencourt P (2015) Prognostic value of $\mathrm{SST} 2$ added to BNP in acute heart failure with preserved or reduced ejection fraction. Clin Res Cardiol 104:491-499 